\title{
TRANSITIONAL FLOW STRUCTURE ON CLASSIC AND WAVY WINGS AT LOW REYNOLDS NUMBERS
}

\author{
I. Zverkov, V. Kozlov, and A. Kryukov \\ Khristianovich Institute of Theoretical and Applied Mechanics \\ Siberian Branch of the Russian Academy of Sciences \\ 4/1 Institutskaya Str., Novosibirsk 630000, Russia
}

\begin{abstract}
The paper presents experimental results on the boundary-layer structure and laminar-turbulent transition on a wavy wing designed for unmanned air vehicles (UAVs). The study has been performed at the chord Reynolds number of the order of $10^{5}$ to compare the wings with smooth and wavy surfaces at similar conditions. The flow past Z-15-25 airfoil was investigated with a particle-image velocimetry (PIV). The method made it possible to obtain a spatial pattern of velocity perturbations on the wing with wavy surface.
\end{abstract}

\section{INTRODUCTION}

Miniaturization of electronic equipment resulted in development of small-scale UAVs. Currently, such UAVs are widely used for both military and civil purposes. Recent researches have been devoted to UAVs with the total weight below $0.5 \mathrm{~kg}$ and the chord Reynolds number (Re) ranging from $10^{4}$ to $10^{5}$. Despite the progress achieved in this field, wide application of UAVs is limited by their modest aerodynamic characteristics, since the most important of them as the stalling angle of attack and lift-to-drag ratio can degrade significantly due to the changes in the boundary layer at the relatively small Reynolds numbers.

Usually, the so-called low and high Reynolds-number regions are selected to be below and above $\operatorname{Re}=10^{6}$, respectively. These regions are different in the structure of the boundary layer on the leeward side of wings. At $\operatorname{Re}>10^{6}$ for the airfoils with the maximum thickness of $12 \%-20 \%$ at about $25 \%$ of the chord length, the laminar-turbulent transition occurs without flow separation in the vicinity of the minimum static pressure. On the contrary, at $\operatorname{Re}<10^{6}$, the laminar boundary layer usually separates, then the laminar-turbulent transition

This is an Open Access article distributed under the terms of the Creative Commons Attribution License 4.0, which permits unrestricted use, distribution, and reproduction in any medium, provided the original work is properly cited. 


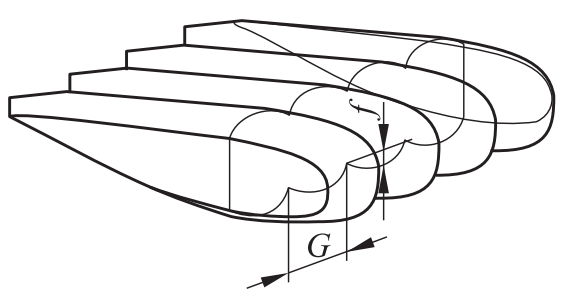

Figure 1 Parameters of a wavy wing: $f$ is the hump height and $G$ is the hump spanwise spacing takes place and, finally, the turbulent boundary layer reattaches forming a local 'transitional' separation zone (separation bubble). The smaller the Reynolds numbers, the larger the effect of the bubble on the wing drag. Theoretical description and computation of the transitional separation bubbles and corresponding laminarturbulent transition is a long standing problem. Its key issues are discussed, e. g., in recent monographs $[1,2]$.

An approach to control the boundary-layer separation and improve aerodynamic characteristics of wings is their shaping. In particular, a spanwise periodic waviness of the lifting surface seems promising. Hereinafter, an unmodified wing is referred to as the classic one, while a wing with wavy surface is denoted as the wavy one.

In a number of publications [3-7], it has already been demonstrated that the wavy surface can increase the stalling angle of attack at low Reynolds numbers. However, the studies show that only certain types of the waviness provide the desired effect. In [8], it was noted that the wavy surface efficiency increases below the Reynolds number of $10^{6}$ depending on such parameters as the hump height $f$ and the hump spanwise spacing $G$ (Fig. 1).

The best results have been obtained at $f=0.03$ and $G=0.11$ of the chord. Moreover, even at lower parameters $(f=0.013$ and $G=0.12)$, some benefits of the wavy wing were found in [9]. In the last work, it was shown that the aerodynamic characteristics of airfoils of different types experience various changes. For example, an increase of both the stalling angle of attack and the maximum lift force was reported for an airfoil with the maximum thickness at $15 \%$ of the chord length, while an aerodynamic hysteresis vanished for the airfoil with the maximum thickness at $25 \%$.

Actually, the studies devoted to the structure of the boundary layer and the conditions that improve the aerodynamic characteristics of wings are scarce so that many questions are still open. In Fig. 2, one can see oil-film visualization images combined with colored contour plots of amplitude distribution of the streamwise disturbance velocity in the boundary layer of classic and wavy wings according to the results presented in [6]. It is clearly seen that both the structure of the flow and the location of the laminar-turbulent transition differ significantly along the hump and groove centerlines. When the transition determined as the position of the disturbance amplitude maximum is at $45 \%$ of the chord along the groove centerline, the flow is not completely turbulent even at $74 \%$ of the chord along the hump centerline. Then, the questions are: what is the flow structure between the hump and the groove and how one can determine or estimate the 


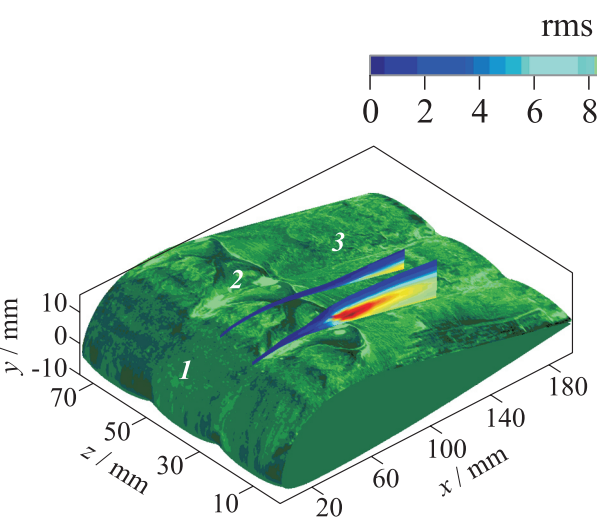

(a)

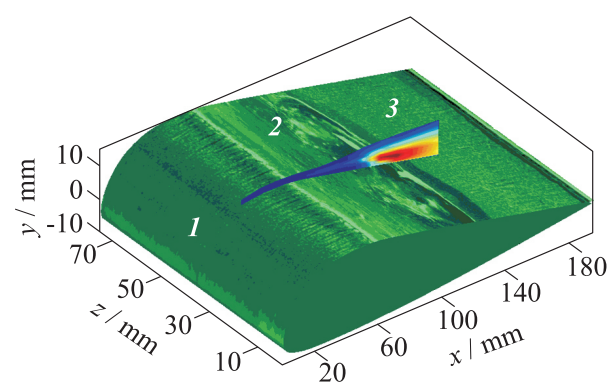

(b)

Figure 2 Oil-film visualization and rms amplitudes of velocity disturbances on classic $(a)$ and wavy $(b)$ wings with profile R-III-A [6]; $\mathrm{Re}=1.5 \cdot 10^{5}$, free-stream turbulence level $\mathrm{Tu}=0.04 \%$, and angle of attack $\alpha=0^{\circ}: 1$ - laminar boundary layer; 2 separation region; and 3 - reattached turbulent flow

spanwise position of the transition? To clarify these issues, the experiments described below have been carried out.

\section{EXPERIMENTAL TECHNIQUES}

The wind tunnel TUG located in a division of the German Aerospace Center (DRL) in Göttingen was used to perform the experiments. It is of open-loop type with a closed test section of 0.3 -meter width and 1.5-meter height, the free-stream turbulence level is about $\mathrm{Tu}=0.15 \%$ (Fig. 3).

A Z-15-25 airfoil with the wing chord $c=297 \mathrm{~mm}$ and the span $l=300 \mathrm{~mm}$ was used in the tests. The details of the airfoil geometry can be found, e.g., in [10]. Surface clutter due to reflection of the laser beam from the test model makes a problem at the PIV measurements in a thin boundary layer. To reduce the reflection, the models were made of transparent acrylic plastic (Fig. 4). The main frame was constructed from glued pieces of acryl and a 'skin' was made of "Vivak" plastic [11]. The parameters of waviness varied slightly from those used in $[6,9]$ being $G=0.097$ and $f=1 \%$ of the chord and the humps extended over $90 \%$ of the chord. The frame and the skin were glued with PFL-100 PU adhesive. The surface roughness was provided less than $0.01 \mu \mathrm{m}$, while the airfoil shape relative error was less than $0.2 \mathrm{~mm}$ for $100 \mathrm{~mm}$.

The models of classic and wavy wings were tested at zero angle of attack and the chord Reynolds number equal to $1.14 \cdot 10^{5}$. In what follows, a chord-aligned 

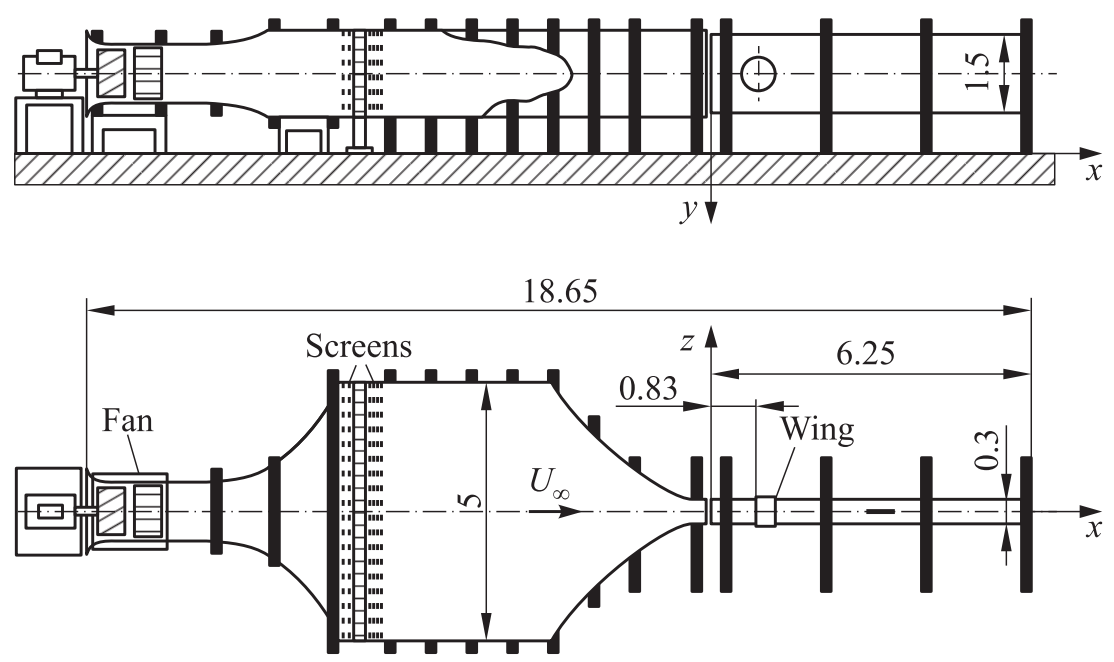

Figure 3 Sketch of the wind tunnel. Dimensions are in meters

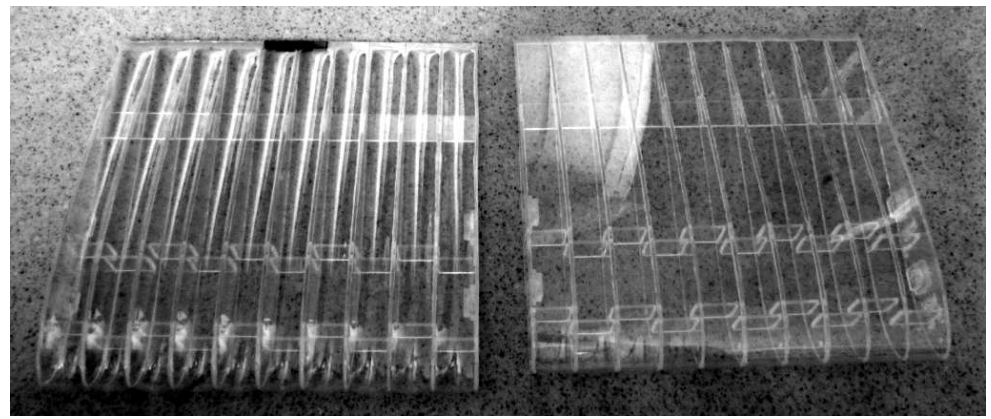

(a)

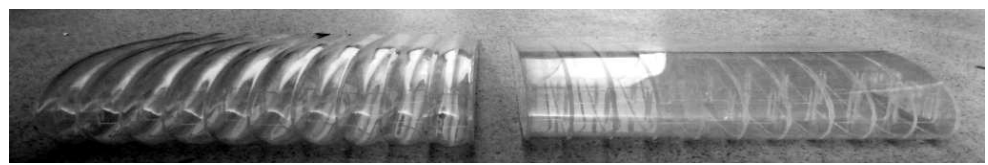

(b)

Figure 4 Test models of the classic (right) and wavy (left) wings: (a) top view; and $(b)$ front view 
Cartesian coordinate system is used with its origin at the wing leading edge in the middle of the span.

The experimental setup is illustrated in Fig. 5 and, in more detail, in Fig. 6. The planar PIV measurements were performed over both the top and the bottom surfaces of the wing (corresponding to the Z-15 and Z-25 shapes, respectively) at different spanwise locations starting from the hump centerline up to the groove centerline with the step of $1 \mathrm{~mm}$. Such measurement procedure was taken in accordance with the previous studies [6] indicating that the flow pattern is symmetrical in respect to the hump and groove centerlines.

The flow was seeded with microparticles of rape-seed oil. These particles were produced in a particle generator with compassed air in Laskin nozzle [12] and were of the size of $100-300 \mu \mathrm{m}$. The particles were used to seed the space of the channel's test chamber. The experiments have been performed as follows. A computer-controlled traversing mechanism moved the Pad 1 (see Fig. 6) $1 \mathrm{~mm}$ at a time in one direction along the model span. The Pad was used as a support for a laser, an optic system to produce the laser sheet, and a CCD (chargecoupled divice) camera installed on the Pad 2. The sheet was oriented in the $(x, y)$-plane and propagated through the model. The camera was focused on the laser-sheet plane whose thickness was about $0.5 \mathrm{~mm}$. A set of PIV data was acquired with the help of shifting the pad along the span. The scanning step along $z$ axis was $1 \mathrm{~mm}$.

In Fig. 7, the surface profile along the span and the $(x, y)$-planes chosen for further analysis are shown. To be able to record the boundary-layer flow between the humps, the frame plane was turned by $12.5^{\circ}$. In this case, the depth resolution was provided to be enough to record the flow inside the entire boundary layer. The PIV data in the area from $10 \%$ to $25 \%$ of the chord for the planes $z=27$ and 30 were rejected because they were spoiled by the laser sheet reflection from the wing rib. For the classic wing, the data were processed using the same procedure.

To increase the resolution, the size of the CCD camera frames was selected to cover only about one four of the chord's length. Hence, four overlaped sets of the PIV data were used to cover the flow along the chord length. To move the camera in the streamwise direction, a computer-controlled traversing mechanism of the Pad 2 was used. The measurement area was divided into three sections along $x$ axis: the first one from $10 \%$ to $40 \%$, the second one from $40 \%$ to $60 \%$, and the third one from $60 \%$ to $100 \%$ of the chord length. The rms velocity perturbations distributions obtained in this regions were used in the postprocessing stage to produce smooth rms velocity perturbations in the whole region. To interpolate the data, the least square technique was used. The distance to the wall was determined using geometrical markers. The accuracy of positioning was estimated to be 0.5 and $0.1 \mathrm{~mm}$ in $x$ and $y$ directions, respectively. The laser pulse duration of $200 \mathrm{~ns}$ was used during the measurements, while the time lag between two images was $75 \mu \mathrm{s}$. For each section, 200 images with 0.5-hertz 


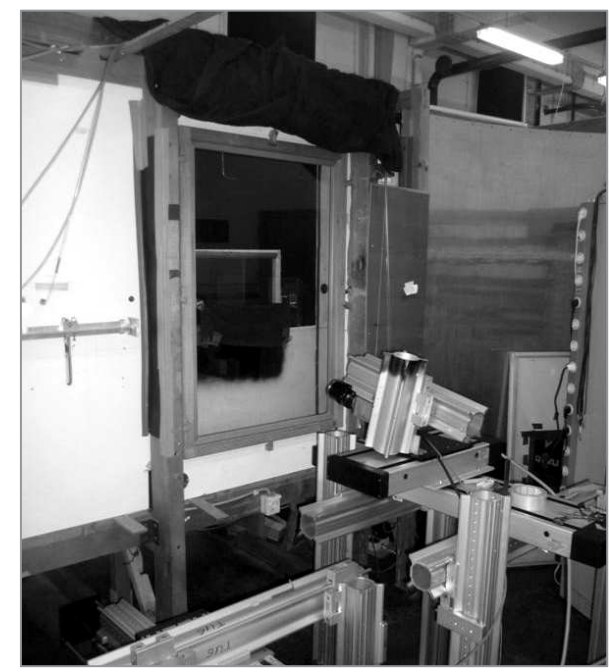

Figure 5 Experimental setup, general view

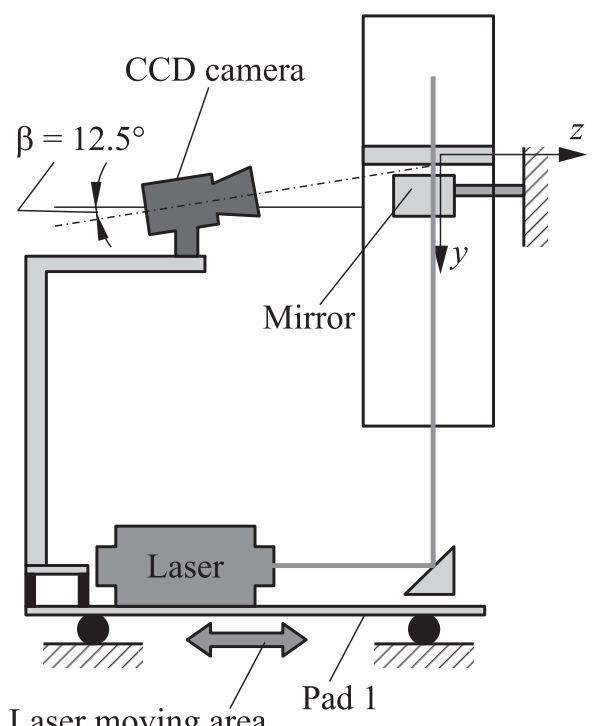

(a)

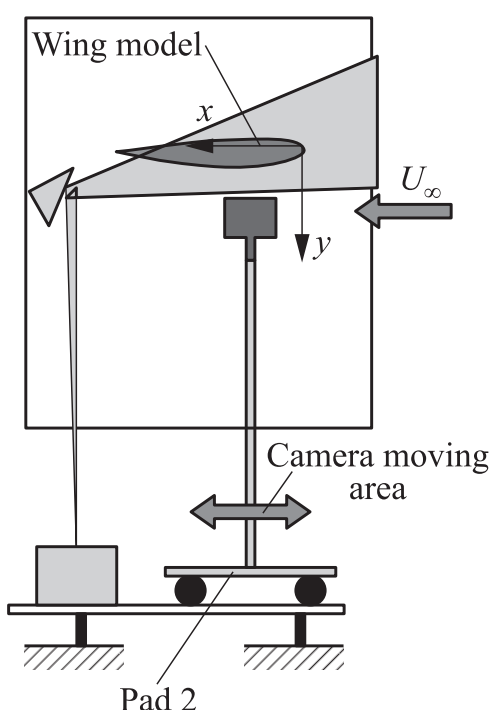

(b)

Figure 6 Scheme of the PIV-measurements: (a) front view; and (b) side view 


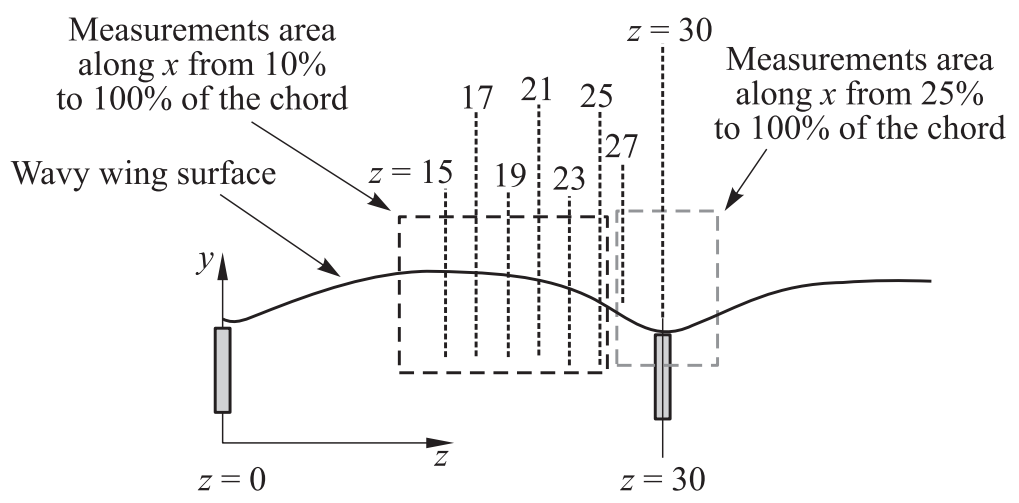

Figure 7 Positioning of measurement planes relative to the hump

frequency frame rate were recorded. These images were used to determine the mean velocity and the rms amplitude of disturbances inside the boundary layer. The data were processed with PIVview2C software bundle kindly provided by the DLR. To determine the velocity distribution, a two-image cross-correlation technique was applied. The details of the method can be found in [13].

\section{WIND TUNNEL DATA}

Different shapes of the top and bottom sides of the test models enable to trace the laminar-turbulent transition depending on the streamwise pressure distribution. The transition location can be estimated with several criteria. One of them used in the present study is the streamwise position of the maximum amplitude of velocity perturbations. Another indication is the variation of the shape factor $H_{12}(x)$ as the ratio of the displacement to the momentum thickness. A sharp increase of $H_{12}$ testifies to boundary-layer separation triggering the laminar flow breakdown, while downstream of the turbulent reattachment, the shape factor goes down again.

The behavior of the disturbance amplitude and the shape factor $H_{12}$ for the classic wing is given in Fig. 8. As the flow was essentially two-dimensional, the results are shown for the plane of symmetry only. The stremwise velocities obtained after a postprocessing of the PIV data were used to calculate the rms values of perturbations and the shape factor $H_{12}$. The maximum velocity perturbations are found in the rear part of the separation bubble. This is in a general qualitative agreement with the hot-wire data reported in [6]. However, the present PIV measurements indicate that the maximum values of the 

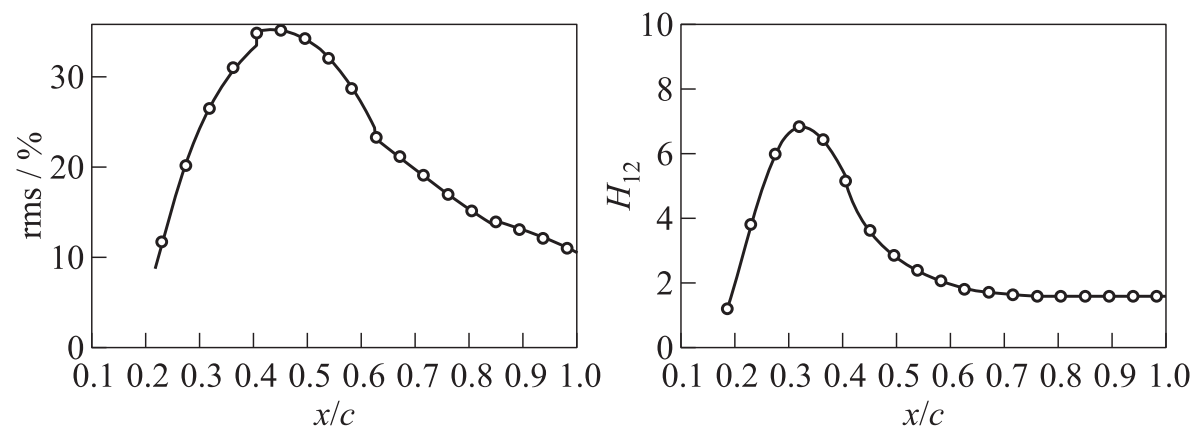

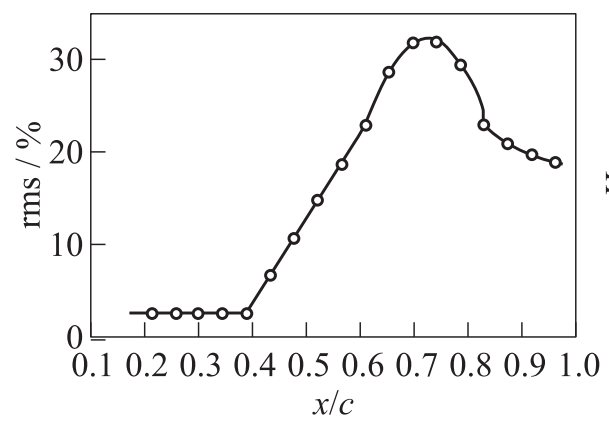

(a)

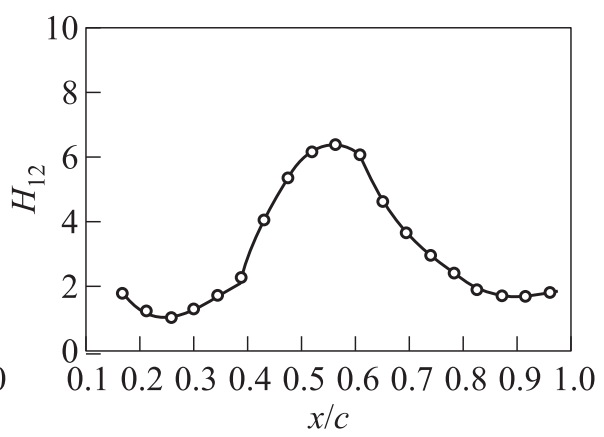

(b)

Figure 8 Streamwise variations of the rms amplitude of velocity perturbations $(a)$ and the shape factor $H_{12}(b)$ at the top (upper row) and bottom (lower row) sides of the classic wing

disturbance amplitude are somewhat different from those observed in [6]. This difference can be explained, probably, by low shooting frequency and a small number of recorded frames compared to the corresponding hot-wire sampling parameters. This, however, is not an obstacle to determine the chordwise locations of the maximum rms values which take place at $45 \%$ and $75 \%$ of the chord for the top and bottom sides, respectively.

Similar data for the wavy wing are presented in Figs. 9 and 10. As seen in Fig. 9, the laminar-turbulent transition along the groove centerline on the top side of the wing is found behind the flow separation, whose occurrence is indicated by the rapid increase of $H_{12}$. The flow turbulization is accompanied by the boundary-layer reattachment so that $H_{12}$ drops. On the top side, for which the maximum airfoil chamber is at $15 \%$ of the chord length, the $H_{12}$ maximum is located at about $20 \%$ of the chord length, while the disturbance maximum is located at about $25 \%$ of the chord length. Hence, the laminarturbulent transition occurs at about $25 \%$ of the chord length along the groove centerline. The separation bubble region is extended to the hump slopes to the 


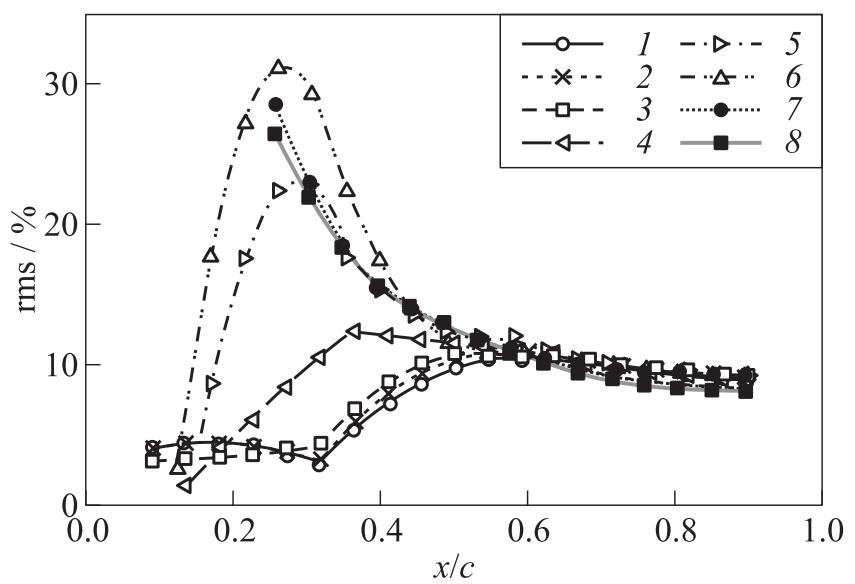

(a)

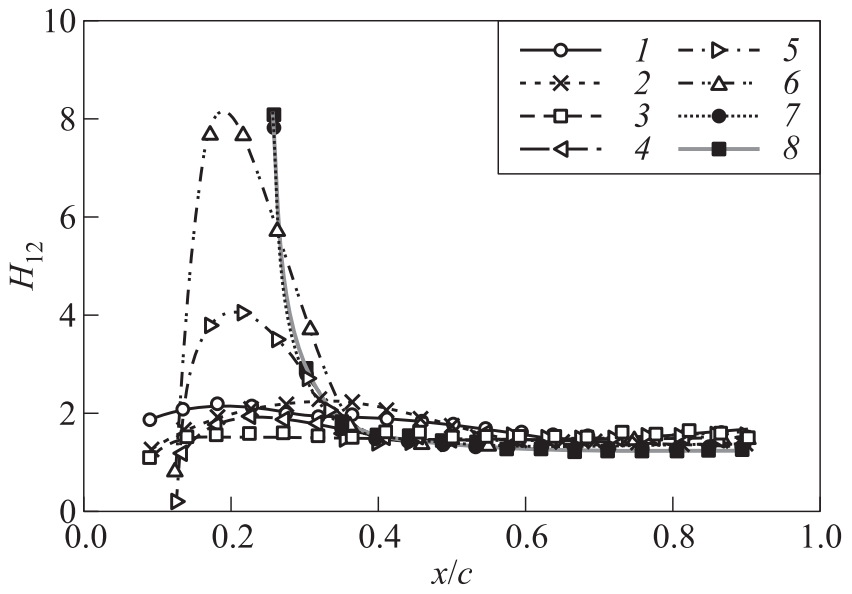

(b)

Figure 9 Streamwise variations of the rms amplitude of velocity perturbations $(a)$ and the shape factor $H_{12}(b)$ at the top side of the wavy wing in different $z$-sections: $1-z=15 \mathrm{~mm} ; 2-17 ; 3-19 ; 4-21 ; 5-23 ; 6-25 ; 7-27$; and $8-$ $z=30 \mathrm{~mm}$

distance up to $5-7 \mathrm{~mm}$ from the groove centerline. The disturbance amplitude and $H_{12}$ curves for $z=23,25$, and 27 are similar to those for $z=30$ (groove centerline), but the maximum values are lower.

At about $30 \%$ of the chord length, the flow reattachment occurs along the hump centerline. This is confirmed by the value of $H_{12}$ which does not grow downstream. The flow can be considered laminar up to $35 \%$ of the chord length as 


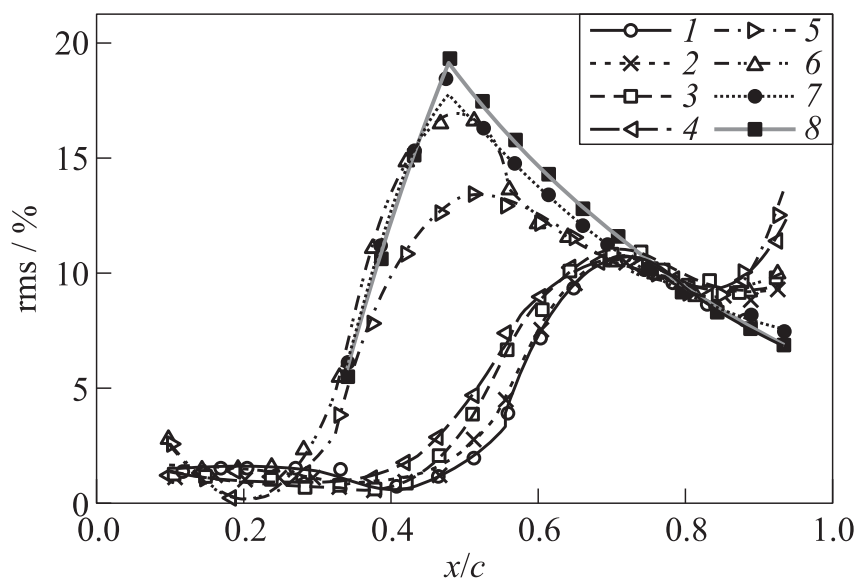

(a)

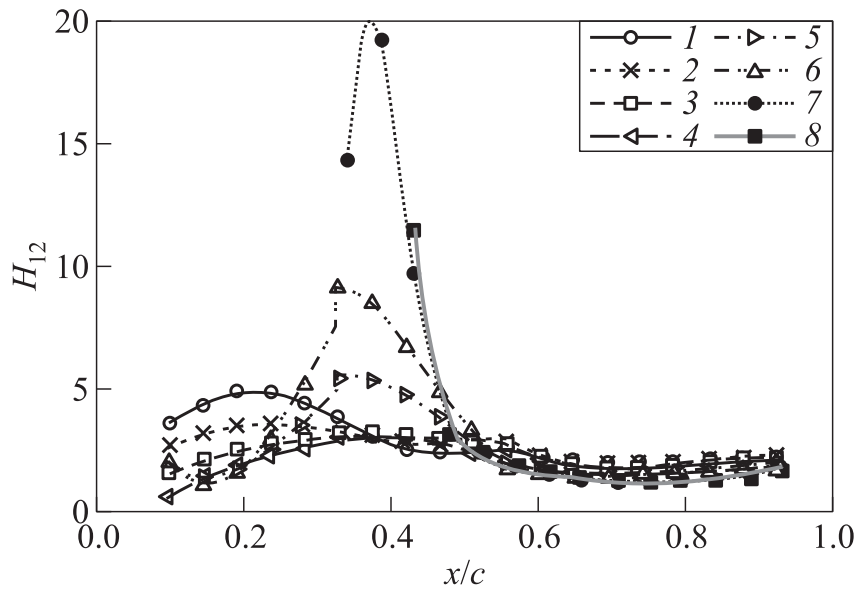

(b)

Figure 10 Streamwise variations of the rms amplitude of velocity perturbations $(a)$ and the shape factor $H_{12}(b)$ at the bottom side of the wavy wing in different $z$-sections: $1-z=15 \mathrm{~mm} ; 2-17 ; 3-19 ; 4-21 ; 5-23 ; 6-25 ; 7-27$; and $8-z=30 \mathrm{~mm}$

the disturbance amplitude shows no significant change in respect to the upstream values at $10 \%$ of the chord length. In the region from $35 \%$ to $58 \%$ of the chord length, the disturbance amplitude grows to $10 \%$, while the value of $H_{12}$ decreases slightly. Further downstream, the turbulence level along the hump and groove centerlines shows quite similar decrease. Hence, the transition location along the hump centerline is estimated to be at about $58 \%$ of the chord length. The 
parameters of the boundary layer located $4-6 \mathrm{~mm}$ aside the hump centerline behave similarly.

For the bottom side of the wing, the maximum camber is located at $25 \%$ of the chord length. In general, the transition looks similar on the top and the bottom sides; however, it is shifted downstream in the last case (compare Figs. 9 and 10). Along the groove centerline, the laminar-turbulent transition occurs at about $47 \%$ of the chord length, which has been in a good agreement with the hot-wire data for R-III-A airfoil (see [6]), whose maximum thickness was also at $25 \%$ of the chord length. The disturbance amplitude maximum along the hump is located at about $70 \%$ of the chord length. Therefore, to estimate an average spanwise laminar-turbulent transition location, one has to take, e. g., the arithmetic average between the disturbance amplitude maxima for the aboveindicated side. For top and bottom sides, this reads to be $40 \%$ and $59 \%$ of the chord length, respectively.

To obtain an impression of the laminar-turbulent transition in the wavy wing boundary layer, a set of representative isosurfaces of rms disturbance amplitudes of the streamwise velocity for the top and bottom sides of the wing are shown in Fig. 11. To produce the figures, data from PIVview2C software without a postprocessing were used.

As seen, the velocity disturbances with amplitude of about $15 \%$ are localized only in the groove area where the separation bubble is present (Fig. 11a), while the disturbances with amplitude of about $7 \%$ cover both the groove and the hump areas (Fig. 11b). However, in the groove area, they are located mostly in the separated shear layer and correspond to laminar-turbulent transition in the groove area, while in the hump area, they approach the wing surface and are shifted further downstream. That is there is an apparent 'spreading' of the disturbances in the spanwise direction followed by a complete covering of the whole boundary layer by turbulence.

\section{CONCLUDING REMARKS}

Distributions of the streamwise disturbance velocity were obtained in the classic and wavy wing boundary layers through PIV measurements. Corresponding isosurfaces of the rms amplitudes of the disturbance velocity demonstrate that the laminar-turbulent transition starts between the humps, where the disturbance amplitude maximum is reached inside a separation bubble. As the disturbance amplitude level decreases downstream, the turbulence spreads in the spanwise direction. The performed experiments indicate that shifting the maximum airfoil thickness $10 \%$ chordwise results in $20 \%$ downstream shift of the laminarturbulent transition location. They also demonstrate that the spanwise-averaged location of the laminar-turbulent transition occurs $10 \%-15 \%$ earlier on the wavy wing in respect to the transition location at a corresponding classic wing. 

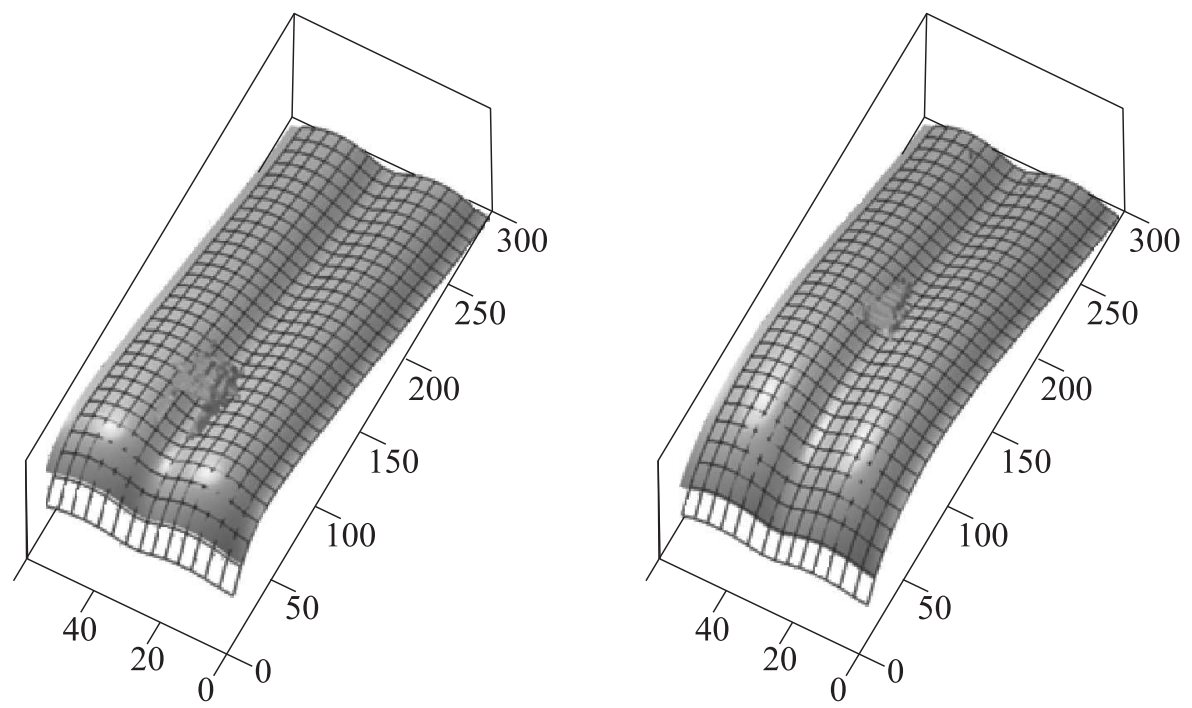

(a)
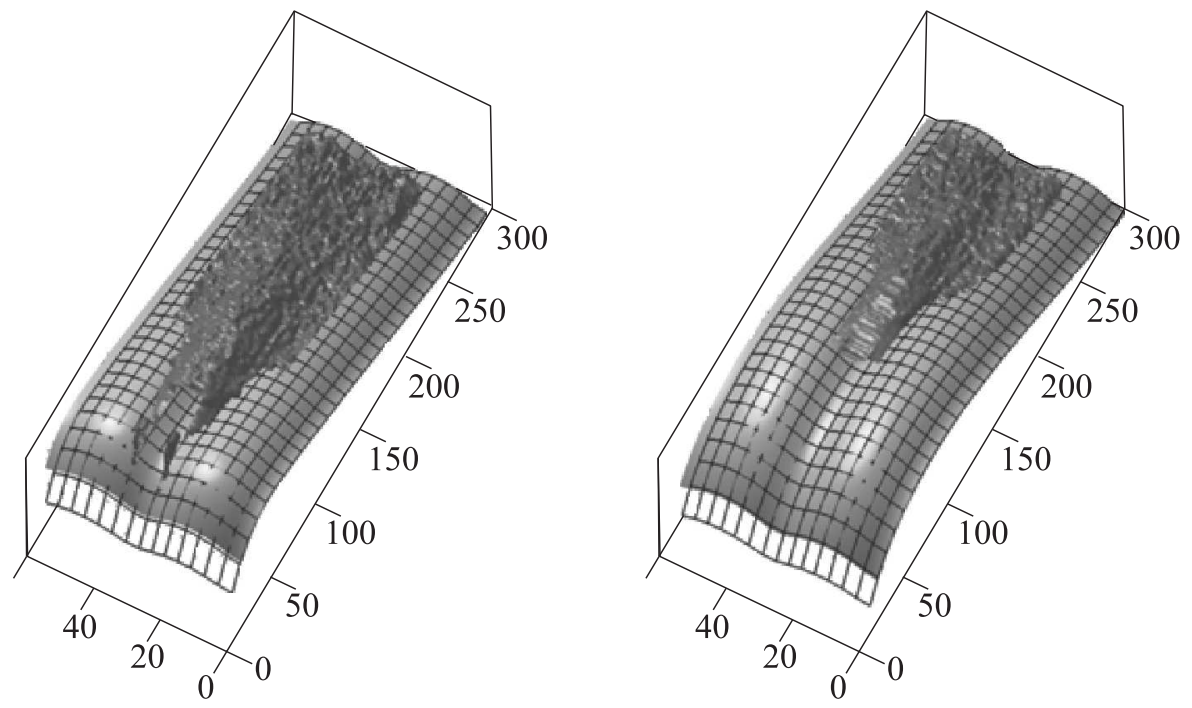

(b)

Figure 11 Isosurfaces of rms disturbance amplitudes in the boundary layer of the wavy wing. Amplitude levels are $15.2 \%$ of $U_{\infty}(a)$ and $7.07 \%$ of $U_{\infty}(b)$ : left column top side and right column — bottom side 
A certain similarity in the near-wall flow impact between the local separation bubbles and vortex generators which are set to prevent a stall on a classic wing takes place. Both form a spanwise periodic structure of the boundary layer consisted of bands with low and high turbulence levels and spanwise altered displacement and momentum loss thicknesses. Such spanwise-periodic boundary layers are more stable in respect to the flow separation than the corresponding spanwise-homogeneous boundary layers. In its turn, these more stable boundary layers display better aerodynamic characteristics, e.g., providing an increase in the stalling angle of attack. However, in contrast to the artificial vortex generators, as the wing angle of attack changes, the local separation bubble adopts its position in a natural way providing in some sense an 'optimal' position for the flow turbulization.

Therefore, in respect to the aerodynamic efficiency, the wavy wings with $f=0.01-0.013$ and $G=0.10-0.12$ of the chord are more preferable than smooth wings for UAVs with chordwise Reynolds number of about $10^{5}$.

\section{ACKNOWLEDGMENTS}

The first author of this paper Ilya Zverkov was supported by the Alexander von Humbold Foundation that is highly appreciated. The investigation was supported by the Russian Foundation for Basic Research (Grants 14-08-00369A, 13-07-00616A, and 13-08-00395A).

\section{REFERENCES}

1. Boiko, A. V., A. V. Dovgal, G. R. Grek, and V. V. Kozlov. 2012. Physics of transitional shear flows: Instability and laminar-turbulent transition in incompressible near-wall shear layers. Dordrecht: Springer. $272 \mathrm{p}$.

2. Shvy, W., Y. Lian, T. Tang, D. Viieru, and H. Liu. 2008. Aerodynamics of low Reynolds number flyers. New York: Cambridge University Press. 177 p.

3. Watts, P., and F.E. Fish. 2001. The influence of passive, leading edge tubercles on wing performance. 12th Symposium (International) on Unmanned Untethered Submersible Technology (UUST) Procceedings. Lee, NH: Autonomous Undersea Systems Inst.

4. Zverkov, I. D., and B. Yu. Zanin. 2003. Wing form effect on flow separation. Thermal Phys. Aeromech. 10(2):197-204.

5. Miklosovic, D. S., M. M. Murray, L.E. Howle, and F. E. Fish. 2004. Leading-edge tubrcles delay stall on humpbuck whale (Megaptera novaeangliae) flippers. Phys. Fluids 16(5):L39-L42.

6. Zverkov, I. D., B. U. Zanin, and V. V. Kozlov. 2008. Disturbances growth in boundary layers on classical and wavy surface wing. AIAA J. 46(12):3149-3158. 
7. Johari, H., C. Henoch, D. Custodio, and A. Levshin. 2007. Effects of leading-edge protuberances on airfoil performance. AIAA J. 45(11):2634-2641.

8. Hansen, K. L., R. M. Kelso, and B.B. Dally. 2011. Performance variations of leading-edge tubercles for distinct airfoil profiles. AIAA J. 46(1):185-194.

9. Zverkov, I., V. Kozlov, and A. Kryukov. 2013. Experimental research of the boundary layer structure at near-critical angles of attack for the classical and wavy wings. Progress in flight physics. Eds. Ph. Reijasse, D. D. Knight, M. S. Ivanov, and I. I. Lipatov. EUCASS advances in aerospace sciences book ser. 5:583-600.

10. Zverkov, I. D., V.V. Kozlov, and A. V. Kryukov. 2011. Influence of wind tunnel freestream turbulence level on boundary layer separation. Thermal Phys. Aeromech. 18(2):213-224.

11. http://www.sheet.bayerpolymers.com/10/Produkte/Vivak.htm (accessed July 1, 2014).

12. Echols, W. H., and J. A. Young. 1963. Studies of portable air-operated aerosol generators. NRL Report 5929.

13. Raffel, M., C.E. Willert, S. T. Wereley, and J. Kompenhans. Particle image velocimetry. A practical guide. Berlin: Springer. 448 p. 